\title{
Obesity and Metabolic Surgery Society of India (OSSI) Recommendations for Bariatric and Metabolic Surgery Practice During the COVID-19 Pandemic
}

\author{
Sandeep Aggarwal ${ }^{1}$ (D) $\cdot$ Kamal Mahawar ${ }^{2} \cdot$ Manish Khaitan $^{3} \cdot$ Praveen Raj $^{4} \cdot$ Randeep Wadhawan $^{5}$. \\ NandaKishore Dukkipati ${ }^{6} \cdot$ Kuldeepak S Kular $^{7}$. Arun Prasad ${ }^{8}$. Aparna Govil Bhasker ${ }^{9}$ Vandana Soni ${ }^{10}$.

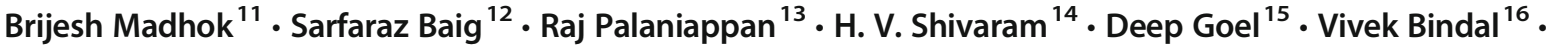 \\ Sukhvinder Saggu ${ }^{17} \cdot$ Rajesh Shrivastava $^{18} \cdot$ Sumeet Shah $^{19} \cdot$ Shrihari Dhorepatil $^{20} \cdot$ Rajesh Khullar $^{21}$
}

Received: 26 May 2020 / Revised: 14 August 2020 / Accepted: 17 August 2020 / Published online: 22 August 2020

(C) Springer Science+Business Media, LLC, part of Springer Nature 2020

\begin{abstract}
Bariatric and metabolic surgery (BMS), the only effective option for patients with obesity with or without comorbidities, has been stopped temporarily due to the ongoing novel corona virus disease (COVID-19) pandemic. However, there has been a recent change in the governmental strategy of dealing with this virus from 'Stay at Home' to 'Stay Alert' in many countries including India. A host of health services including elective surgeries are being resumed. In view of the possibility of resumption of BMS in near future, Obesity and Metabolic Surgery Society of India (OSSI) constituted a committee of experienced surgeons to give recommendations about the requirements as well as precautions to be taken to restart BMS with emphasis on safe delivery and high-quality care.
\end{abstract}

Keywords Bariatric surgery $\cdot$ COVID-19 $\cdot$ Coronavirus $\cdot$ Resumption $\cdot$ Pandemic

\section{Background}

The COVID-19 pandemic has resulted in postponement of all planned elective operations. Unfortunately, bariatric and metabolic surgery (BMS) has taken the worst brunt of the pandemic. Most centers have put a complete halt to new referrals and operations. Obesity is associated with several comorbidities including type 2 diabetes mellitus (T2DM), hypertension, and obstructive sleep apnea (OSA) [1]. Patients with obesity have an impaired immune response resulting in enhanced risk of various infections including COVID-19 [2]. Worryingly, the severity of COVID-19 is worse in patients suffering from obesity $[3,4]$. As the BMS has been deferred due to the COVID-19 pandemic, the patients with obesity continue to suffer from its adverse consequences [5-7].

Sandeep Aggarwal

sandeep_aiims@yahoo.co.in

Extended author information available on the last page of the article
In view of the above and considering obesity and bariatric surgery to be of equal importance as other chronic lifethreatening diseases and cancer, the Obesity and Metabolic Surgery Society of India (OSSI) decided to frame recommendations which will help bariatric surgeons to prepare for the possible recommencement of bariatric surgery in the near future. These recommendations are predominantly based on the opinion of experts given the paucity of data on this subject. The published recommendations by several national and international societies were also taken in account while compiling these [8-11].

\section{Resuming BMS-Safety First}

Patients with obesity have a higher risk of morbidity/ mortality should they develop COVID-19 infection [3, 4]. This will hold true for the perioperative period too as the patient is still obese in the immediate perioperative period [12]. Thus, the aim of these recommendations is to accord the highest priority to the safety of patients and healthcare workers $(\mathrm{HCW})$ while resuming BMS. This 
will mandate the induction of safety measures in all standard operating procedures (SOPs) involving the journey of the patient from the pre-admission phase to discharge from the hospital as well as post-discharge care.

It is generally accepted that resumption of BMS, like any elective surgery, will require a sustained decrease in new COVID-19 cases in the community as a prerequisite $[5,8]$. Therefore, resumption of bariatric surgery must be done cautiously based on national, state, and local health authority policies. There should be sufficient availability of resources including testing for COVID-19, personal protective equipment (PPE), and health personnel.

\section{Patient Selection}

Emergency surgeries including those for bleeding, perforation, and acute small bowel obstruction need to be performed urgently with all precautions including a full personal protective equipment (PPE). Similarly, some conditions including vomiting due to anastomotic strictures, internal hernias causing symptoms, and gastric band erosion may require an early-urgent/semi-urgent surgery [13]. For non-urgent elective surgery, to restart the practice, patient deemed to be less complex should be selected. Younger patients with few comorbidities may be ideal. Initially, it may be best to avoid patients more than 60 years, those with a body mass index of $50 \mathrm{~kg} / \mathrm{m}^{2}$ or more, complex revisional bariatric operations, and highrisk patients including those with severe obstructive sleep apnea (OSA), history of venous thromboembolism (VTE), moderate to severely impaired pulmonary function, history of significant heart disease, cirrhosis, and end-stage renal disease $[7,14]$.

\section{Preoperative Preparation of a Patient}

\section{Before Admission}

Preoperative patient counselling and evaluation should be done using telemedicine protocols. The counselling should include the discussion about COVID-19-related risk. All investigations including tests for COVID-19 must be complete before admission. The patient should be admitted a day prior to surgery and the pre-arrival swab should be done $48-72 \mathrm{~h}$ prior to date of admission. All patients should preferably self-isolate for 2 weeks before the operation. Good control of comorbidities is desirable especially in patients with diabetes mellitus, hypertension and OSA.

Pre-Arrival Screening should be done to include a detailed COVID-19-related history and history of fever, cough, travel/contact, and other suspicious symptoms as per the government and hospital protocols.

COVID-19 Testing Testing for COVID-19 should be guided by the national and state health policies. The reverse transcriptase-polymerase chain reaction (RT-PCR) should be done. All patients should have negative results for the COVID-19 antigen test $48-72 \mathrm{~h}$ before the operation. The tests should be done preferably prior to admission for surgery.

\section{At Admission}

The patient should be admitted a day prior to the surgery to decrease the hospital stay. At reception area, the patient and attendant body temperature should be screened using the non-contact method, and surgical masks should be provided. The patient should be admitted in an isolation room with only one attendant and reevaluated thoroughly by the operating team. All HCW should always wear a mask.

Imaging If required, imaging should be focused on portable radiographs and bedside portable ultrasound/ POCUS - point-of-care ultrasonography - to avoid unnecessary patient transport. Chest computed tomography (CT) scan is a useful screening method [15] and can be done routinely or selectively in high-risk patients, e.g., those with pre-existing cardio-respiratory comorbidity including but not limited to severe OSA, coronary artery disease, pulmonary hypertension, bronchial asthma, and interstitial lung disease. CT should be done preferably in the morning of the surgery or a day prior to further reduce the risk of operating on patients with active infection.

\section{Consent}

The consent form should be modified to include the following salient points:

- Patient is willing to get operated while the pandemic situation has not subsided.

- Hospital will not be held responsible if patient develops COVID-19 after surgery.

- Patient has been explained that they may have a more severe disease and increased risk of mortality should they develop COVID-19 after surgery.

A consent form specially modified for surgery during the COVID-19 pandemic has been included in Appendix 1.

\section{Indoor and Operating Room Protocols}

General guidelines about hand hygiene, physical distancing, cough etiquettes, and cleaning and disinfection protocols are 
well known and should always be adhered to. All healthcare personnel must be assessed and monitored daily. The HCW should be regularly updated about the latest protocols.

Elective bariatric operations should ideally be carried out on a cold, COVID negative site. If such a separate hospital is not available, then all elective operations should be carried in a different area/wing of the main hospital. New operating room (OR) SOPs regarding COVID-19 should be created and nursing, anaesthesia, surgery checklists revised. The operating team should allow for longer-than-usual time per case (add 50\% to 75\% of total theatre time required) for adequate checks to be performed, PPE donning and doffing safely, and cleaning the OR space in between cases. Although it is desirable to consider changing the pressure inside the OR from positive to negative [16], a suitable alternative is to switch off the positive pressure $30 \mathrm{~min}$ before induction and start 30 min after sanitization at the end of the surgery since positive pressure is a feature in most ORs [17].

\section{Operating Room Personnel}

All the staff in the OR should be asymptomatic and ideally tested regularly for COVID-19 based on national/ state/local guidelines. They should work exclusively in a non-COVID OR. They should undergo fit testing for filtering face piece 3 (FFP3) masks and should be trained in donning and doffing of PPE using videos or online demonstrations. Risk assessment for the appropriate use of PPE should be done. However, any PPE should include the following:

- FFP3 mask/N 95 respirator mask

- Eye protection — goggles or face shield. For longer cases, a respirator hood

- Fluid proof shoe cover

- Fluid resistant double gown

- Double gloves

\section{Anaesthesia Protocols}

Endotracheal intubation is a high-risk procedure for cross transmission both to the anaesthetist and patient. The number of personnel in the OR during the intubation procedure should be limited. Detailed guidelines about such protocols are available in literature [18, 19]. The surgical and nursing teams should enter $15 \mathrm{~min}$ after intubation although Yeo et al. has recommended this time interval to be $3 \mathrm{~min}$ based on an air recycling rate of 25 to 30 cycles per hour [20].

\section{Surgical Protocols}

There should be a minimum number of staff in the OR, and everyone should wear PPE. There is some concern that the surgeons may be out of practice. So, it may be important to develop procedure-specific "time out" checklists that reduce errors and ensure that surgeons finish all steps of the operation. In the beginning, it may be worth two trained surgeons pairing up to facilitate surgery and reduce chances of errors and complications.

Although other viruses have been demonstrated in surgical smoke [21], there is no evidence at present that smoke can transmit COVID-19. However, the absence of evidence at present does not mean that transmission cannot occur. Thus, even at the cost of erring on the side of being overcautious, all practical measures for prevention of exposure to surgical plume must be followed. These include avoidance of open technique for pneumoperitoneum, the use of by muscle splitting optical trocar for entry into obese abdomen, use of balloon ports, reduction of the intra-abdominal pressure to as low as possible without compromising surgical exposure or patient safety, careful handling of ports, and keeping the electrosurgery settings to minimum possible $[10,11]$. Some of the precautions required specially in BMS are listed in Table 1.

Smoke evacuation systems with filtration mechanism can be used to reduce the risk of infection from surgical plume. Several commercial devices are available, and it is recommended to procure one such system during the preparation period. One extra $5-\mathrm{mm}$ port can be used for suction cannula to suck simultaneously during energy usage in the absence of commercial smoke evacuation systems.

At the end of the procedure, the pneumoperitoneum should be completely evacuated safely, and only then the trocars should be removed. The same precaution is required during the extraction of the specimen (if any) or conversion to open procedure. Surgical drains and nasogastric tubes should be avoided. If used, the drain should be clamped until complete evacuation of gas. Port closures must be done carefully in deflated obese

Table 1 Suggested additional precautions during bariatric and metabolic surgery

- Leaks around Nathanson Liver Retractor during surgery need to be minimized by additional purse string skin sutures when needed

- Surgeon should ensure careful and swift introduction of staplers and suture material to reduce gas leaks

- The gastric calibration tube should be handled with all precautions that were taken during intubation. 
abdomen and if need be, incision extended slightly to ensure a proper closure.

In case of robotic surgery, the robotic surgeon at console should also wear basic PPE and take precautions. The team should include experienced surgeons who can handle leakage and smoke evacuation beside the patient.

\section{Postoperative Care}

The surgical team should adhere to standardized enhanced recovery after bariatric surgery (ERABS) protocols for early discharge [22]. Strict VTE prophylaxis is indicated as there is a greater risk of complications such as deep vein thrombosis (DVT) in patients with COVID-19 [23]. In case of the patient developing cough, fever, or breathlessness after surgery, testing for COVID-19 and/or CT may be needed to confirm or exclude this dreaded possibility. Diet and exercise do not require any special modifications beside general advice on immunity boosting diet, vitamin D, vitamin C, and zinc-rich food/supplements. There may be difficulty in procuring supplements; hence, importance of nutritious home food should be stressed. Low-intensity home exercises and yoga can be suggested due to closure of gymnasiums and fitness centers during the lockdown.

\section{Discharge Protocols}

Patients should be discharged as early as possible to "only discharge at home" as the default pathway. General COVID-19 prevention advice including social distancing and the use of mask should be reiterated. The patient should be taught about COVID-19-related symptoms and signs and those suggestive of surgical complications. Any respiratory symptoms in the postoperative period should be treated urgently, and COVID-19 pneumonia must be considered in the differential diagnosis. Patients should be provided an emergency $24 \times 7$ contact number. Patients should call the operating surgeon directly for any problem rather than going through their own general practitioner. Regular video interactions with patients should be scheduled to ensure safe recovery.

\section{Reporting of Data to OSSI}

OSSI encourages all bariatric surgeons in India to submit data regarding any serious adverse events (SAE) as soon as possible following resumption of bariatric surgery. A SAE form with separate questions for COVID-19 will be created and put on the OSSI website soon.

\section{Conclusion}

The COVID-19 pandemic in India is still spreading, and the crisis is evolving. As such, it is not possible for OSSI to predict any time frame for resumption of bariatric surgery. It may be prudent to initiate BMS when the pandemic curve shows a deceleration in a region. The local, state and national advisories/guidelines should be adhered to for resumption of surgery and rational use of resources. The safety of patients and HCW is extremely important, and therefore, all suggested measures regarding patient and HCW screening, PPE, and OR protocols should be adopted to minimize transmission of COVID-19 during BMS. As the COVID-19 situation is dynamic, these recommendations may be revised according to the evolving situation as well as the collected data over next $3-$ 6 months.

\section{Compliance with Ethical Standards}

Conflict of Interest The authors Sandeep Aggarwal, Kamal Mahawar, Manish Khaitan, Praveen Raj, Randeep Wadhawan, NandaKishore Dukkipati, Kuldeepak S Kular, Arun Prasad, Aparna Govil Bhasker, Vandana Soni, Brijesh Madhok, Sarfaraz Baig, Raj Palaniappan, H.V. Shivaram, Deep Goel, Vivek Bindal, Sukhvinder Saggu Rajesh Shrivastava, Sumeet Shah, Shrihari Dhorepatil, Rajesh Khullar declare no conflict of interest.

Ethical Approval Statement This article does not contain any studies with human participants or animals performed by any of the authors.

Informed Consent Statement Informed consent does not apply.

\section{Appendix 1}

\section{Bariatric surgery and COVID-19 outbreak}

\section{Information sheet}

We produced this information sheet to provide patients undergoing bariatric surgical procedures with additional information on the risks of undergoing anaesthesia and surgery around the coronavirus (COVID-19) outbreak.

It is very important that you notify your medical team of any risks you may have had of being exposed to coronavirus. These include (but are not limited to contact with people who have been ill in the last two weeks and underlying medical conditions.

As with any surgical procedure, there are benefits and risks to undergoing general anaesthesia and surgery. However, there are additional risks related to the COVID-19 outbreak that we need you to be aware of. This will ensure that you are giving your informed consent to undergoing your procedure. 
Please read the below carefully and add your initials in the boxes to confirm you understand the information you have read and been given.

Patient

Initials

I have read and understand the following additional risks of undergoing general anaesthesia and surgery during the COVID-19 outbreak. These include (but are not limited to):

- It is possible that I am an asymptomatic (not showing symptoms at present) carrier of coronavirus.

- My undergoing a general anaesthetic may be associated with development of)pneumonia (chest infection) due to coronavirus.

- If I develop pneumonia due to coronavirus this may lead to respiratory failure(serious difficulty in breathing) which may require respiratory support in a critical care setting (e.g. intensive care unit (ICU)).

- This may result in deterioration in my condition, which may result in death.

This request and consent for surgery is being signed by me with the full understanding of the information contained above in this document, based on my review of it and my discussions with my medical team who have answered my questions to my satisfaction.

Patient Name :

Patient Signature :

Staff Name:

Staff Signature:

Designation:

Date:

\section{References}

1. Andolfi C, Fisichella PM. Epidemiology of obesity and associated comorbidities. J Laparoendosc Adv Surg Tech A. 2018;28(8):919 24. https://doi.org/10.1089/lap.2018.0380.

2. Ahn S-Y, Sohn S-H, Lee S-Y, et al. The effect of lipopolysaccharideinduced obesity and its chronic inflammation on influenza virusrelated pathology. Environ Toxicol Pharmacol. 2015;40:924-30.

3. Simonnet A, Chetboun M, Poissy J, et al. High prevalence of obesity in severe acute respiratory syndrome coronavirus-2 (SARS$\mathrm{CoV}-2$ ) requiring invasive mechanical ventilation. Obes Silver Spring Md. 2020;28:1195-9.

4. Richardson S, Hirsch JS, Narasimhan M, et al. Presenting characteristics, comorbidities, and outcomes among 5700 patients hospitalized with COVID-19 in the New York City area. JAMA. 2020;323(20):2052-9.
5. Bhasker AG, Greve JW. Are patients suffering from severe obesity getting a raw deal during COVID-19 pandemic? Obes Surg. 2020. https://doi.org/10.1007/s11695-020-04677-z.

6. Hussain A, Mahawar K, El-Hasani S. The impact of COVID-19 pandemic on obesity and bariatric surgery. Obes Surg. 2020;30(8): 3222-3. https://doi.org/10.1007/s11695-020-04637-7.

7. Executive Council of ASMBS. Safer through surgery: American Society for Metabolic and Bariatric Surgery statement regarding metabolic and bariatric surgery during the COVID-19 pandemic [published online ahead of print, 2020 Jun 6]. Surg Obes Relat Dis. 2020;S1550-7289(20)30318-X. doi:https://doi.org/10.1016/j. soard.2020.06.003

8. Yang W, Wang C, Shikora S, et al. Recommendations for metabolic and bariatric surgery during the COVID-19 pandemic from IFSO. Obes Surg. 2020;30:2071-3.

9. Joint Statement: roadmap for resuming elective surgery after COVID-19 pandemic [Internet]. Am. Coll. Surg. [cited 2020 
May 23]. Available from: https://www.facs.org/covid-19/clinicalguidance/roadmap-elective-surgery

10. Francis N, Dort J, Cho E, et al. SAGES and EAES recommendations for minimally invasive surgery during COVID-19 pandemic. Surg Endosc. 2020;34(6):2327-31. https://doi.org/10.1007/ s00464-020-07565-w.

11. Srivastava A, Nasta AM, Patahnia BS, et al. Surgical practice recommendations for minimal access surgeons during COVID 19 pandemic- Indian inter society directives. J Min Access Surg 2020;16(3):195-200.

12. Aminian A, Kermansaravi M, Azizi S, et al. Bariatric surgical practice during the initial phase of COVID-19 outbreak. Obes Surg. 2020;30:3624-7.

13. Rubino F, Cohen RV, Mingrone G, et al. Bariatric and metabolic surgery during and after the COVID-19 pandemic: DSS recommendations for management of surgical candidates and postoperative patients and prioritisation of access to surgery. Lancet Diabetes Endocrinol. 2020;8:640-8.

14. Daigle CR, Augustin T, Wilson R, et al. A structured approach for safely reintroducing bariatric surgery in a COVID-19 environment published online ahead of print. Obes Surg. 2020. https://doi.org/ 10.1007/s11695-020-04733-8.

15. Ai T, Yang Z, Hou H, et al. Correlation of chest CT and RT-PCR testing in coronavirus disease 2019 (COVID-19) in China: a report of 1014 cases. Radiology. 2020;296:E32-40.

16. Chow TT, Kwan A, Lin Z, et al. Conversion of operating theatre from positive to negative pressure environment. J Hosp Infect. 2006;64:371-8.

17. Coccolini F, Perrone G, Chiarugi M, et al. Surgery in COVID-19 patients: operational directives. World J Emerg Surg. 2020;15(1):
25. Published 2020 Apr 7. https://doi.org/10.1186/s13017-02000307-2.

18. Cook TM, El-Boghdadly K, McGuire B, et al. Consensus guidelines for managing the airway in patients with COVID -19: guidelines from the Difficult Airway Society, the Association of Anaesthetists the Intensive Care Society, the Faculty of Intensive Care Medicine and the Royal College of Anaesthetists. Anaesthesia. 2020;75:785-99.

19. Malhotra DN. COVID operation theatre-advisory and position statement of Indian Society of Anaesthesiologists (ISA national). Indian J Anaesth. 2020;64(4):259-63. https://doi.org/10.4103/ija. IJA_288_20

20. Yeo D, Yeo C, Kaushal S, et al. COVID-19 \& the general surgical department-measures to reduce spread of SARS-COV-2 among surgeons. Ann Surg. 2020;272:e3-4.

21. Kwak HD, Kim SH, Seo YS, et al. Detecting hepatitis B virus in surgical smoke emitted during laparoscopic surgery. Occup Environ Med. 2016;73:857-63.

22. Fantola G, Nagliati C, Foletto M, et al. Is there a role for ERAS program implementation to restart bariatric surgery after the peak of COVID-19 pandemic? Obes Surg. (2020). https://doi.org/10.1007/ s11695-020-04676-0.

23. Liu Z, Zhang Y, Wang X, et al. Recommendations for surgery during the novel coronavirus (COVID-19) epidemic. Indian J Surg. 2020;82:124-8. https://doi.org/10.1007/s12262-020-021733.

Publisher's Note Springer Nature remains neutral with regard to jurisdictional claims in published maps and institutional affiliations. 


\section{Affiliations}

\section{Sandeep Aggarwal ${ }^{1}$ (D) Kamal Mahawar ${ }^{2}$ - Manish Khaitan ${ }^{3} \cdot$ Praveen Raj $^{4} \cdot$ Randeep Wadhawan $^{5}$. NandaKishore Dukkipati ${ }^{6} \cdot$ Kuldeepak S Kular $^{7}$ • Arun Prasad ${ }^{8}$. Aparna Govil Bhasker ${ }^{9}$. Vandana Soni ${ }^{10}$. Brijesh Madhok ${ }^{11}$ - Sarfaraz Baig ${ }^{12}$ - Raj Palaniappan ${ }^{13} \cdot$ H. V. Shivaram ${ }^{14}$. Deep Goel ${ }^{15}$. Vivek Bindal $^{16}$.

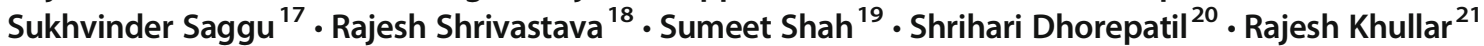

\author{
Kamal Mahawar \\ kmahawar@gmail.com \\ Manish Khaitan \\ khaitanmanish11@gmail.com \\ Praveen Raj \\ praveenraj@me.com \\ Randeep Wadhawan \\ randeepwadhawan@yahoo.com \\ NandaKishore Dukkipati \\ Kishoredukkipati@gmail.com \\ Kuldeepak S Kular \\ drkskular@gmail.com \\ Arun Prasad \\ surgerytimes@gmail.com \\ Aparna Govil Bhasker \\ draparnagovil@gmail.com \\ Vandana Soni \\ vandan_soni@yahoo.com \\ Brijesh Madhok \\ brijesh.madhok@nhs.net
}

1 Department of Surgical Disciplines, All India Institute of Medical Sciences, New Delhi, India

2 Sunderland Royal Hospital, Sunderland, UK

3 KD Hospital, Ahmedabad, India

4 Gem Hospital \& Research Centre, Coimbatore, India

5 Manipal Hospitals, New Delhi, India

6 KIMS Livlife Centre, Hyderabad, India

7 Kular Hospital P. Ltd., Khanna, India

8 Apollo Hospitals, New Delhi, India

Gleneagles Global Hospital, Mumbai, India

10 Max Institute of Laparoscopic, Endoscopic and Bariatric Surgery Max Super Specialty hospital, New Delhi, India

11 University Hospitals of Derby \& Burton NHS Foundation Trust, University of Nottingham (School of Medicine), Nottingham, UK
Sarfaraz Baig

docsarfarazbaig2@gmail.com

Raj Palaniappan

docraj@me.com

H. V. Shivaram

hvshivaram@gmail.com

Deep Goel

goel_deep@me.com; drdeepgoel12@gmail.com

Vivek Bindal

bindal.vivek@gmail.com

Sukhvinder Saggu

drsagguss@gmail.com

Rajesh Shrivastava

dr.rajeshshree70@gmail.com

Sumeet Shah

sumeetshah01@gmail.com

Shrihari Dhorepatil

sdhorepatil@gmail.com

Rajesh Khullar

drajeshkhullar@gmail.com

12 Digestive Surgery Clinic, Belle Vue, Kolkata, India

13 Apollo Hospitals, Chennai, India

14 Aster CMI Hospital, Bangalore, India

15 BLK Super-Speciality Hospital, New Delhi, India

16 Institute of Minimal Access, Metabolic and Bariatric Surgery (iMAS), Sir Ganga Ram Hospital, New Delhi, India

17 Apollo Spectra Hospital, New Delhi, India

18 Shreeji Hospital, Vadodara, India

19 Max Smart Super Speciality Hospital, New Delhi, India

20 Shree Hospital, Pune, India

21 Max Institute of Laparoscopic, Endoscopic and Bariatric Surgery Max Super Specialty hospital, New Delhi, India 